\title{
IDENTIFICATION OF THE (DETERMINISTIC OR RANDOM) NATURE OF THE WHEAT PRICE VARIABILITY WITH THE APPLICATION OF RECURRENCE QUANTIFICATION ANALYSIS
}

\author{
Łukasz Pietrych, $\mathrm{PhD}^{1}$ \\ Faculty of Economic Sciences, Warsaw University of Life Sciences - SGGW
}

\begin{abstract}
In this article performed was an analysis of the time series which presents the wheat price by means of recurrence quantification analysis. The main research problem was to ascertain whether the time series for this agricultural raw material may be considered as a system in which chaos has the deterministic nature, possibly with slight random disturbances. It was proved that it is justified to believe that the time series under analysis is characterized with the occurrence of deterministic chaos. The literature review led to believe that although this issue had been analysed in a large number of papers, most specialists confirm there is no conclusive evidence of chaos in economic or financial data.
\end{abstract}

Keywords: deterministic chaos, recurrence quantification analysis, wheat prices JEL codes: C2, E31

\section{INTRODUCTION}

Due to progress in exact sciences available became new mathematical and statistical tools. Therefore, economists eventually notice that many economic phenomena cannot be explained with the so-called traditional approach based upon the linear analysis. The reductionist approach, used in the traditional economic theory, often neglected the relations or reciprocal interactions among the market agents and their effect upon economic systems on the macroeconomic scale. This approach was aimed at simplifying questions, and eventually, at looking for analytical solutions. In the economic literature more and more often underlined is not only the concept that economies are complex adaptive systems consisting of a large number of components, but also their inter- relations (Faggini and Lux, 2009). Mitchell emphasizes that complex system investigations were aimed at explaining in an interdisciplinary way the process of arising of adaptive behaviours in complex systems consisting of a large number of simple components which are not subject to central control, and among which numerous interactions will occur (Mitchell, 1998).

The necessity of complying with the necessity of developing more realistic models gave rise to new concepts and research tools in economic sciences. A new research perspective, taking into consideration non-linear dynamic systems functioning in the economy, causes considerable changes in the main current of the economic theory. Economists began to discern the functioning of certain patterns in the economy by recognizing its complexity.

${ }^{1}$ Corresponding author: Nowoursynowska 166,02-787 Warsaw, Poland, lukasz_pietrych@sggw.pl, +4822 5934148 
The purpose of this article was to ascertain whether the system under analysis might be treated as one in which chaos has deterministic nature, possibly with slight random disturbances. The investigations were focused on the agricultural; market, and precisely, upon the price variability of winter wheat. Be stressed that although many papers have been already dedicated to this issue, this research issue is still significant and remains unambiguous. This article constitutes a contribution to the literature, since empirical investigations have been complemented with the agricultural market.

\section{THEORETICAL BACKGROUND}

Theoretical foundations of neoclassical economic theory originate from the postulates developed by Adam Smith and the school of utilitarianism, represented mainly by J.S. Mill. Basing upon the developed assumptions of market behaviours, the neoclassical economic theory comprised a concept which brings one closer to conceive the functioning of all markets in the economy, incl. financial markets. Therefore, basing upon this neoclassical perspective there have been developed, among others, financial theories of competitive equilibrium, like the effective market hypothesis and random walk theory (Velasquez, 2010).

While investigating into financial markets, there were detected, however, certain behaviours contrary to the neoclassical theory. The pertinent literature shows some evidence for the occurrence of season anomalies, excessive variability in time series, correlation of returns on assets and company size, market indicators or seasons of the year. Such investigations have been enumerated, among others, by Guimaraes, Kingsman and Taylor (1989) as well as by Loan and MacKinlay (1999). The conclusions arising therefrom can indicate that the prices are not in equilibrium, investors do not act as reasonable individuals and the market changes cannot be described through the random walk theory. This means that the neoclassical concept does not describe correctly the actual behaviour of financial markets (Velasquez, 2010).

In the references underlined is the idea that the discrepancy among the economic and financial the- ory and reality is attributable to the fact that the neoclassical economic theory reminds of the Newtonian physics (Mirowski, 2001). For example, it is assumed that the agent which is a scaled model of the entire society has extraordinary possibilities in the field of gaining processing of information. This assumption is dubious for the heterogeneity of individuals and their cognitive skills, limited to various extents. Secondly, the neoclassical economic theory was limited to investigating only into systems in equilibrium, neglecting the dynamic nature of certain economic phenomena. As it was proved soon, the equilibrium paths - even in the case of standard economic models - allow one to conceive the issue under analysis more thoroughly, since actually the dynamic economic system may fail to achieve the state referred to as the point of equilibrium, and it may end in limit cycles or even in chaotic paths of highly irregular type. Finally, economists preferred linear models or at least models transformable to a linear form through linearization. Such models, however, do not regard properly the asymmetry of such phenomena, like depressions or periods of recession, price bubbles on stock exchanges with related crunches or the occurrence of regular and irregular business cycles. Such behaviours in the neoclassical economic theory were perceived as anomalies (Faggini and Lux, 2009).

In consequence, the neoclassical economic theory cannot be translated into empirical observations, and the accepted assumptions do not seem reasonable. Thus, the failure of the neoclassical economic theory is believed to be embedded in the selected way of discourse and the set of research tools (Velasquez, 2010). An alternative to economic research becomes more and more often the concept presented by the theory of chaos with pertinent research tools. As Zbilut has it, so far there has been done much research aimed at finding whether economic time series may be modelled with methods which take into consideration the non-linear chaotic dynamics (Zbilut, 2005). Basing upon observations of real life economic systems it may be concluded that on the one hand they seem chaotic, but on the other they are generated by a certain form of determinism. Here, as examples may be given share prices on financial markets, economic growth, price cycles of agricultural raw materials, 
business cycles or overlapping generation models for currency crises (Creedy and Martin, 1994).

\section{MATERIALS AND METHODS}

For the specific nature of economic data (short time series, non-stationarity and the occurrence of white noise), in the literature - as an alternative to the traditional analysis based upon mathematical chaos models - postulated is the application of a relatively simpler analytical method, viz. recurrence quantification analysis (RQA). The idea of recurrence as the fundamental feature of many dynamic systems was introduced by Poincaré in 1890 (1890). In the successive century recorded was a considerable progress in the theory of dynamic systems. In 1987, Eckmann et al. introduced the method of recurrence plots (RP) in order to visualize the recurrence of states in the phase space for dynamical systems (Eckmann et al., 1987). Those plots are a graphical representation of the following relation (Marwan et al., 2002):

$$
R_{i, j}=H\left(\varepsilon-\left\|X_{i}-X_{j}\right\|\right), i, j=1, \ldots, M
$$

where:

$X_{i}, X_{j}$ - states in space $R^{m}$;

$M \quad$ - number of states;

$H \quad$ - Heaviside step function $H(x)=1$, if $x \geq 0$; $H(x)=0$, if $x<0$;

$\|X\| \quad-$ norm of vector $\mathrm{X}$ in space $R^{m}$ (usually, this is the Euclidean or a normal norm);

$\varepsilon \quad-$ the so-called cut-off parameter (non-negative real number).
If the points $X_{i}$ and $X_{j}$ belonging to a certain phase space are separated from each other by no more than $\varepsilon$, then $R_{i, j}=1$, which is marked as a black point; otherwise, $R_{i, j}=0$ (a white point). Consequently, the base of the plot described by equation (1) is a $(0,1)$ zero-one square matrix $R_{M M}$. According to the nature of the process under analysis, the points on the plot constitute structures having different forms. In 1992, Zbilut and Webber proposed a quantitative procedure for determining standards with statistic measures, and named this approach RQA. Initially, Zbilut and Webber introduced 5 variables to quantify standards in the plots. The references for recurrence plots and their interpretations are shown in Table 1.

The aforementioned quantitative measures supply information on various properties of RP and simultaneously they complement one with another. In order to determine them applied was the software Visual Recurrence Analysis (VRA). The empirical material was the time series of weekly winter wheat prices quoted on the Warsaw Commodities Exchange S.A. (WGT). Those data were downloaded from the website e-WGT. The period under analysis lasted from 20.04.2006 to 26.04.2018. The total number of observations utilized was 629 .

\section{RESULTS AND DISCUSSION}

Before proceeding to making recurrence plots, described was the structure of the time series under analysis by means of a graph, histogram and descriptive statistics measures. The amplitude oscillations

Table 1. RQA indices and their interpretation

\begin{tabular}{|l|l|}
\hline RQA index & \multicolumn{1}{c|}{ Interpretation } \\
\hline$\%$ REC & recurrence rate - the percentage ratio of recurrence states (black points in the diagram) to all states \\
\hline$\%$ DET & $\begin{array}{l}\text { The percentage of recurrence points which form linear segments parallel to the plot diagonal. \%DET } \\
\text { is the measure of degree of determinism }\end{array}$ \\
\hline MAXLINE & Periodic signal to determine the long segments of a line, whereas short lines mean chaos \\
\hline TREND & $\begin{array}{l}\text { This measure determines the drift and non-stationarity of the phenomenon under analysis (a value } \\
\text { close to 0 means the stationarity of a series, whereas a negative value - the occurrence of a trend }\end{array}$ \\
\hline ENT & $\begin{array}{l}\text { A high value of entropy occurs in the case of periodic changes, a low value corresponds to chaotic } \\
\text { systems, and eventually, a very low value (close to } 0 \text { ) - to an uncorrelated noise }\end{array}$ \\
\hline
\end{tabular}

Source: own study on the basis of Marwan et al. (2007). 

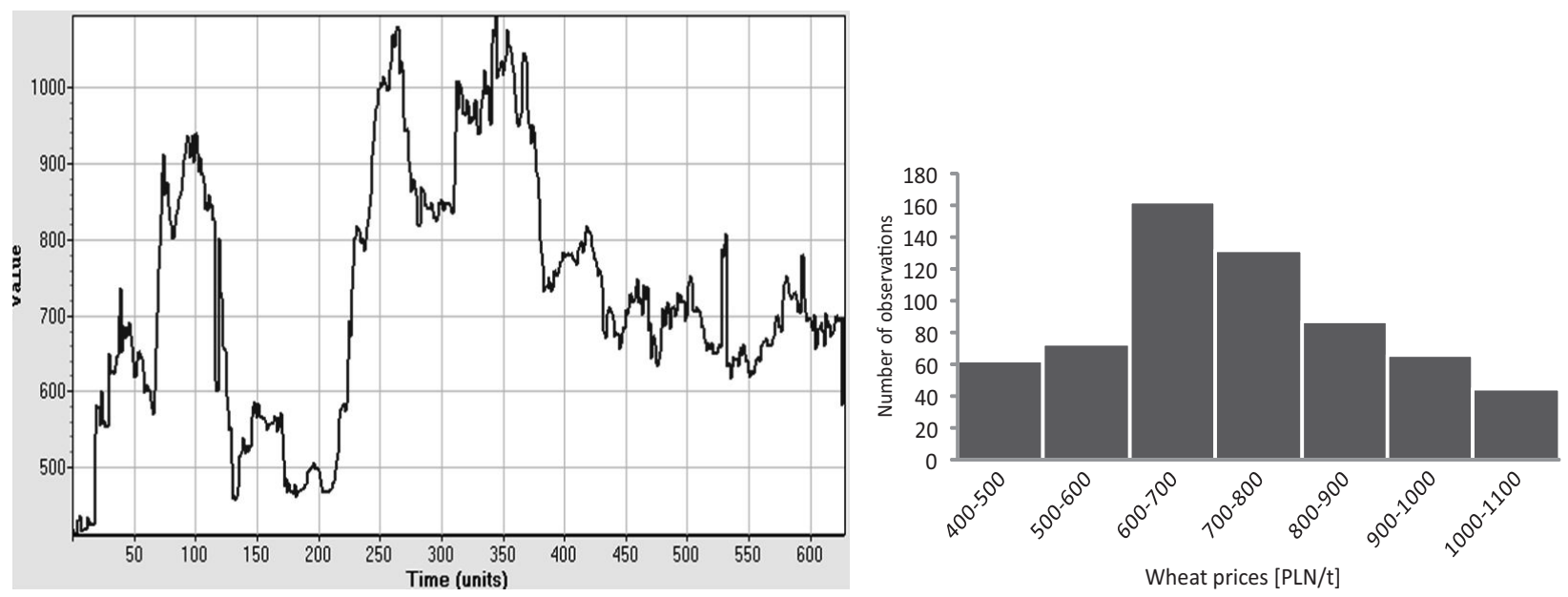

Figure 1. Time series for wheat prices (left-hand side) and size histograms for wheat prices (right-hand side)

Source: own study on the basis of the eWGT data.

for the time series of wheat amounts to PLN 685.44/ /tonne. Low values of the variability coefficient imply either a small or average variability of the time series under analysis. Recorded was a right-side asymmetry of distribution, which corresponds with the histogram shown on Figure 1 (right-hand side).

The empirical significance level viz. the probability of gaining the given ADF value assuming the genuine nature of zero hypothesis $\mathrm{p}$ is high - approx. 0.41 in the case of time series of wheat prices and 0.59 . Therefore, there are no reasons for rejecting the hypothesis that the series under analysis is nonstationary. Nevertheless, be stressed that in the case of economic data from a period of several years, the condition of stationarity for the time series is usually impossible to be satisfied.

In the subsequent phase, the investigations were divided into two stages. In the first part, RQA measures were determined for the entire time series; then, the series was divided into sub-periods and calculated were the measures for each of them (those periods are referred to as epochs). For the investigations' stake it was assumed that the length of each epoch is equal to 250 observations and its regular change takes place every 50 observations, which actually means that each epoch overlaps with the subsequent one with 200 observations. Generally speaking, assuming that is the length of each epoch $d_{e}$ and stands for the shift, in the time series the shift extremities correspond with the days starting at $t=(i-1) d_{e}+1$ and ending at $t=(i-1) d_{e}+N_{e}+1$. On the one hand, the application of such an approach enables a global scale investigation into changes, and on the other - an analysis of changes within the entire time series by making calculations for various epochs (Fabretti and Ausloos, 2005).

In the RQA assumed were the following embedding parameters: $\tau=$ delay $=9 m, m=$ embed $=8$, $\varepsilon=1.5$, which means that the relation (1) assumes the following form: $R_{i, j}=H\left(1.5-\left\|X_{i}-X_{j}\right\|\right)$. More information on the issue of phase space reconstruction can be found in (Takens, 1981). Instead, one of the most frequent methods used for determining time delay $d$, presented in the pertinent literature is Average Mutual Information Function (AMI), proposed by Freser and Swinneya (1986). In the case of the embedding dimension $m$ proposed was the method False Nearest Neighbors (FNN), developer by Kennel et al. (1992). The values of RQA parameters calculated in various epochs and for the entire period are shown in Table 2.

Chaotic processes are characterized with quite low levels of Shannon entropy and a high degree of recurrence. In the case of the series under analysis, the entropy index initially falls from epoch to epoch, and later on it become stabilized; yet, in this phase it 
Proceedings of the 2018 International Scientific Conference 'Economic Sciences for Agribusiness and Rural Economy' No 2, Warsaw, 7-8 June 2018, pp. 130-135

Table 2. Descriptive statistical for the time series of winter wheat price and Dickey-Fuller test

\begin{tabular}{|l|c|c|c|c|c|}
\hline Measure of descriptive statistics & $\begin{array}{c}\text { Min } \\
\text { (PLN/tonne) }\end{array}$ & $\begin{array}{c}\text { Max } \\
\text { (PLN/tonne) }\end{array}$ & $\begin{array}{c}\text { Variability } \\
\text { coefficient } \\
(\%)\end{array}$ & $\begin{array}{c}\text { Asymmetry } \\
\text { coefficient }\end{array}$ & ADF statistics \\
\hline Time series for the wheat price & 410 & 1095.24 & 22.33 & 0.79 & $\mathrm{p}=0.41$ \\
\hline
\end{tabular}

Source: own study on the basis of the eWGT data.

is still other than zero, which would imply random processes. This fall is particularly discernible in epoch five. As far as the recurrence index is concerned, in compliance with the previous investigations, for Brown motions it is on an level of $18 \%$, instead for the function sinus $-15 \%$ (Fabretti and Ausloos, 2005). Therefore, it is clear that except epoch five, the level of this index falls within this interval; therefore, this corroborates the assumptions that the time series is characterized with chaotic changes. The tabled results of investigations (Table 3 ) reveals well the determinism of the process under analysis which is most conspicuous in the first four epochs. The values of MaxLine, viz. the longest linear segment measured parallelly to the main diagonal on the recurrence plot confirms that chaotic oscillations intensified in the second phase of the period.

The result of ADF test is also corroborated by negative values of TREND index. Be also noticed that the epoch in which noticed are some changes in the dynamic of the time series presented is epoch five, for the observations from 201 to 451. Those observations correspond with the period starting at the beginning of February 2011, when an increase in prices was recorded; therefore, it may be stated that the observations in this period considerably depart from other observations in the phase space.

\section{CONCLUSIONS}

Free market economies seem to be systems rather more dynamically unstable than deterministic in which case episodes of instability may be attributed to external shocks. In such circumstances it is very hard to consider a concept with linear cause and effect relationships, and in consequence, more realistic seems to be the assumption that relations occurring between the economic factors and the variables are non-linear. Hence, it is necessary to do research aimed at revealing the essence of the mechanisms operating in various sectors of economy. The articles presents the results of preliminary investigations into identifying deterministic chaos in economic time series. There have been made investigations aimed at ascertaining the nature (deterministic or random) of the time series under analysis. Nevertheless, the application of RQA does not authorize one to formulate unequivocal conclusions concerning the occurrence

Table 3. RQA indices for wheat prices

\begin{tabular}{|c|c|c|c|c|c|c|c|c|}
\hline \multirow{2}{*}{ RQA index } & \multicolumn{7}{|c|}{ Epoch number } & \multirow{2}{*}{ All period } \\
\hline & 1 & 2 & 3 & 4 & 5 & 6 & 7 & \\
\hline$\%$ REC & 17.78 & 18.82 & 19.40 & 17.95 & 12.49 & 15.32 & 21.14 & 15.20 \\
\hline$\%$ DET & 35.12 & 36.91 & 44.31 & 37.37 & 20.35 & 23.60 & 22.47 & 30.18 \\
\hline Entropy & 2.44 & 2.35 & 2.55 & 2.52 & 1.81 & 1.89 & 1.87 & 2.35 \\
\hline MaxLine & 168 & 212 & 193 & 143 & 70 & 70 & 86 & 212 \\
\hline Trend & -76.10 & -58.98 & -196.11 & -158.63 & -122.35 & -180.18 & -181.70 & -21.33 \\
\hline
\end{tabular}

Source: own study on the basis of the eWGT data. 
of deterministic chaos in the time series under analysis. Those investigations need complementing with more accurate methods, at least with a verification of non-linearity by means of no-parametric methods.

The investigations presented hereunder make a certain contribution to the literature, since empirical research has been complemented with the agricultural market, and precisely the market of cereals. Hitherto investigations used to be focused on attempts at identifying deterministic chaos on financial and currency markets. Thus, it seems reasonable to determine the nature of processes in this sector of economy, viz. in agriculture by applying many methods independent one of another, which so far has been done to a little extent.

\section{REFERENCES}

1. Creedy, J., Martin, V. (1994). Chaos and Non-Linear Models in Economics. Edward Elgar Publishing Limited.

2. Eckmann, J.P., Oliffson Kamphorst, S., Ruelle, D. (1987). Recurrence Plots of Dynamical Systems. Europhysics Letters, 4 (91), pp. 973-977.

3. Fabretti, A., Ausloos, M. (2005). Recurrence plot and recurrence quantification analysis techniques for detecting a critical regime. Examples from financial market indices. International Journal of Modern Physics C, 16 (5), pp. 671-706.

4. Faggini, M., Lux, T. (eds.) (2009). Coping with the complexity of economics. Springer. Italy.

5. Fraser, A., Swinney, H. (1986). Independent coordinates for strange attractors from mutual information. Physical Review A, 33, pp. 1134-1140.
6. Kennel, M., Brown, R., Abarbanel, H. (1992). Determing embedding dimension for phase space reconstruction using a geometrical construction. Physical Review A, (45), pp. 3403-3411.

7. Marwan, N., Carmen Romano, M., Thiel, M., Kurths, J. (2007). Recurrence plots for the analysis of complex systems. Physics Reports, 438, pp. 237-329.

8. Marwan, N., Wessel, N., Meyerfeldt, U., Schirdewan, A., Kurths, J. (2002). Recurrence plot based measures of complexity and its application to heart rate variability data. Physical Review E, 66 (2), pp. 1-8.

9. Mirowski, P. (2002). Machine Dreams: Economics Beyond a Cyborg Science. Cambridge University Press, Cambridge.

10. Mitchell, M. (1998). A Complex-Systems Perspective on the 'Computation vs. Dynamics' Debate in Cognitive Science. SFI Working Paper. Retrieved from: http://web.cecs.pdx.edu/ mm/cogsci98.pdf [Accessed 29.03.2018].

11. Poincaré, H. (1890). Sur la probleme des trois corps et les équations de la dynamique [On the problem of the three bodies and equations of dynamics]. Acta Mathematica, 13, pp. 1-271.

12. Takens, F. (1981). Detecting strange attractors in turbulence. Lecture Notes in Mathematics, 898, pp. 366-381 .

13. Velasquez, T. (2010). Chaos Theory and the Science of Fractals in Finance. Odeon, 5, pp. 230-264.

14. Zbilut, J.P. (2005). Use of Recurrence Quantification Analysis in Economic Time Series. In: Salzano, M., Kirman, A. (eds.) Economocs: Complex Windows. Springer. pp. 91-104.

15. Zbilut, J.P., Webber, C.L. (1992). Embedding and delays as derived from quantification of Recurrence Plot. Physics Letters A (171), pp. 199-203. 